\title{
Utilidad de la telemedicina en las enfermedades respiratorias
}

\section{Utility of telemedicine in respiratory diseases}

\author{
José Luis Sandoval-Gutiérrez,* Sergio Monraz-Pérez,* \\ Rosaura Esperanza Benítez-Pérez, ${ }^{*}$ Eduardo Mireles-Cabodevila ${ }^{\ddagger}$
}

*Instituto Nacional de Enfermedades Respiratorias Ismael Cosío Villegas; ${ }^{\ddagger}$ Respiratory Institute. Cleveland Clinic.

El prefijo «tele» significa «a distancia» o «a lo lejos» en griego. En materia de salud, el intercambio de información a distancia mediante tecnologías de la información y comunicación (TIC) ha adoptado diferentes terminologías a medida que aumenta la oferta de servicios. Es así como los términos telemedicina y telesalud han evolucionado desde la aparición de los primeros servicios de salud a distancia en Estados Unidos y Europa a finales de los años sesenta. ${ }^{1}$

La Organización Mundial de la Salud (OMS) desde el año 2000 ha englobado todas las acciones que impliquen el uso de TIC como cibersalud o e-salud. Definiéndola como el uso costo-eficaz y seguro de las TIC y las comunicaciones en la salud y a los ámbitos relacionados con ella, como inclusión de los servicios de atención, vigilancia y documentación sanitarias, educación, conocimientos e investigaciones en materia de salud. Dentro de los componentes descritos de la cibersalud se encuentran algunos conceptos que se han utilizado de manera intercambiable, pero tienen un ámbito específico de acción, tales como: el expediente médico electrónico, la telemedicina, la mSalud (uso de dispositivos móviles y/o aplicaciones), el aprendizaje a distancia o teleeducación, la educación relacionada con el uso de las TIC y la interoperabilidad o desarrollo de normas, redes y logística necesaria para el establecimiento de la cibersalud. ${ }^{2}$

Por lo tanto, la telemedicina es una rama de la cibersalud que utiliza redes de comunicación para proveer de servicios de cuidados a la salud y/o educación médica desde un punto geográfico a otro, por lo general, en situaciones en que la distancia es un factor crítico ${ }^{3}$ (Figura 1).

Por su parte, la teleeducación (e-learning) es el proceso de formación a distancia en salud basado en el uso de las

Correspondencia:

Dr. José Luis Sandoval-Gutiérrez

Instituto Nacional de Enfermedades Respiratorias Ismael Cosío

Villegas, Ciudad de México, México.

Correo electrónico: sandovalgutierrez@gmail.com

Trabajo recibido: 25-IX-2019; aceptado: 22-X-2019. tecnologías de la información y las telecomunicaciones que posibilitan el aprendizaje interactivo, flexible y accesible a cualquier receptor potencial. ${ }^{4}$

Las enfermedades respiratorias representan un problema de morbimortalidad en el campo de la salud pública, ${ }^{5,6}$ esto debido a que existe subdiagnóstico, además de un pobre seguimiento terapéutico (Tabla 1).

Las características geográficas de nuestro país no permiten que las distancias a centros especializados faciliten la necesaria referencia y contrarreferencia. Los usos de las TIC permiten una adecuada comunicación entre pacientes y médicos, así como apoyo técnico entre profesionales, y tiene el potencial de mejorar la atención médica en regiones desatendidas que cuentan con poca o nula atención de especialistas.

A esta problemática se suma el déficit de especialistas en neumología en México, ${ }^{7}$ por lo que el diagnóstico y tratamiento de las patologías respiratorias se asienta principalmente en el primer nivel de atención. Personal que requiere un esquema de capacitación constante en este rubro y que sería difícil de alcanzar de manera presencial.

En nuestro país, en 1995, se implementó por primera vez en Latinoamérica el uso de la telemedicina en el Centro Médico Nacional 20 de Noviembre del Instituto de Seguridad y Servicios Sociales de los Trabajadores del Estado (ISSSTE). Este sistema permite tener comunicación con siete estados de la república, además de proporcionar un medio de difusión a los eventos académicos que este centro realiza. Después de 24 años este sistema sigue funcionando exitosamente y se ha expandido.

La telemedicina se ha aplicado en diferentes condiciones respiratorias: enfermedad pulmonar obstructiva crónica (EPOC), asma, insuficiencia respiratoria crónica, enfermedades neuromusculares, ventilación domiciliaria, rehabilitación pulmonar, apnea del sueño.

Existen varias oportunidades en las TIC como son el diagnóstico (teleespirometría, telerradiología), monitorización (telemonitoreo de signos vitales), apoyo en la toma de decisiones (teleconferencias, llamadas de segunda opinión espe- 


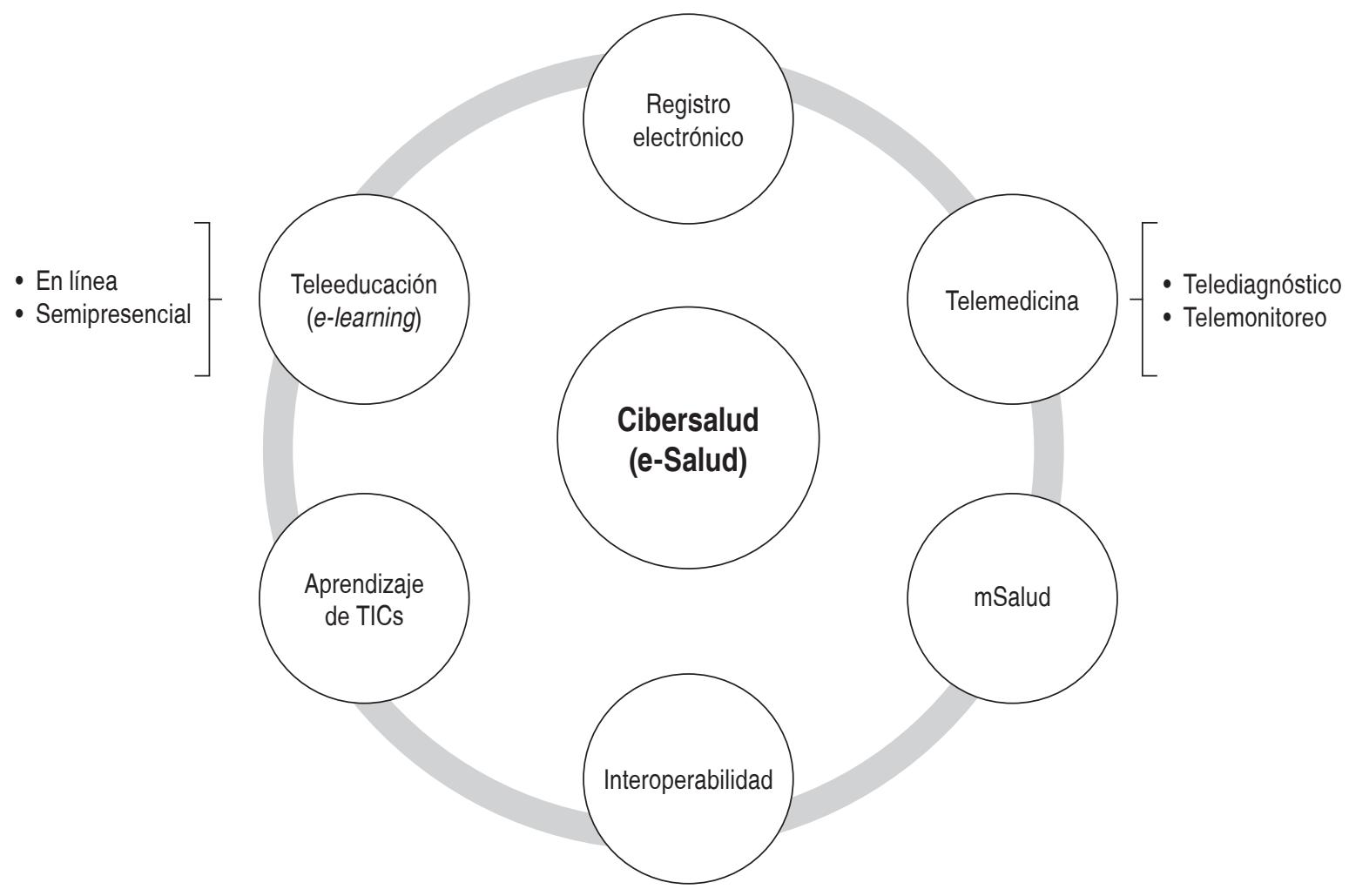

(https://www.gob.mx/salud/cenetec/acciones-y-programas/que-es-la-telesalud-y-la-telemedicina)

Figura 1: Telemedicina y telesalud.

cializada), educación (telementoría, teleevaluación), así como de atención (teleconsulta, teleterapia, telerrehabilitación). ${ }^{8,9}$

El nivel de la tecnología se ha sofisticado lo que permite una adecuada cibersalud. ${ }^{24} \mathrm{~A}$ través del centro de evaluación tecnológica (CENETEC) desde 2004 se ha implementado un programa a nivel nacional en el que hay más de 600 centros que permiten una comunicación fluida entre pacientes y profesionales, lo cual ha llevado a tener una asociación mexicana de telemedicina ${ }^{25}$ y la realización de congresos sobre este tópico. ${ }^{26}$

En la actualidad, el Instituto Mexicano del Seguro Social (IMSS) tiene dentro de su programación la implementación de un sistema TIC para sus derechohabientes y población general. ${ }^{27}$

La nueva generación de médicos denominados millennials ${ }^{28}$ tienen desde su infancia la familiaridad de las nuevas tecnologías cibernéticas, por lo que ya utilizan esta vía a través de sus teléfonos celulares, tabletas electrónicas y computadoras, siendo ésta una enorme área de oportunidad para lograr una interconexión adecuada.

Hay varios puntos a considerar para iniciar un programa de telemedicina exitoso, los cuales pueden resultar inconvenientes como el costo inicial del equipo de hardware y software, falta de conocimiento de los pacientes y de
Tabla 1: Telemedicina en las enfermedades respiratorias.

\begin{tabular}{|c|c|}
\hline $\begin{array}{l}\text { Patología } \\
\text { respiratoria }\end{array}$ & Aplicación de la telemedicina \\
\hline Asma & - Mejoría del control del asma \\
\hline Apnea del sueño & - Diagnóstico y tratamiento ${ }^{12}$ \\
\hline $\begin{array}{l}\text { Enfermedades } \\
\text { neuromusculares }\end{array}$ & $\begin{array}{l}\text { - Reducción de hospitalizaciones y visitas a } \\
\text { urgencias }\end{array}$ \\
\hline EPOC & $\begin{array}{l}\text { - Mejoría de la actividad física }{ }^{15} \\
\text { - Calidad de la espirometría }{ }^{16} \\
\text { - Disminución del uso de recursos } \\
\text { asociados a cuidados de la salud }{ }^{17} \\
\text { - Reducción de costos y admisiones } \\
\text { hospitalarias }{ }^{18} \\
\text { - Reducción de exacerbaciones }{ }^{19} \\
\text { - Reducción en la estancia hospitalaria y } \\
\text { admisiones a urgencias }{ }^{20}\end{array}$ \\
\hline $\begin{array}{l}\text { Rehabilitación } \\
\text { pulmonar }\end{array}$ & $\begin{array}{l}\text { - Rehabilitación pulmonar en EPOC, } \\
\text { reducción de exacerbaciones y } \\
\text { hospitalizaciones }{ }^{21,22}\end{array}$ \\
\hline $\begin{array}{l}\text { Trasplante } \\
\text { pulmonar }\end{array}$ & - Detección temprana de complicaciones ${ }^{23}$ \\
\hline
\end{tabular}




\section{Centros}

espirométricos

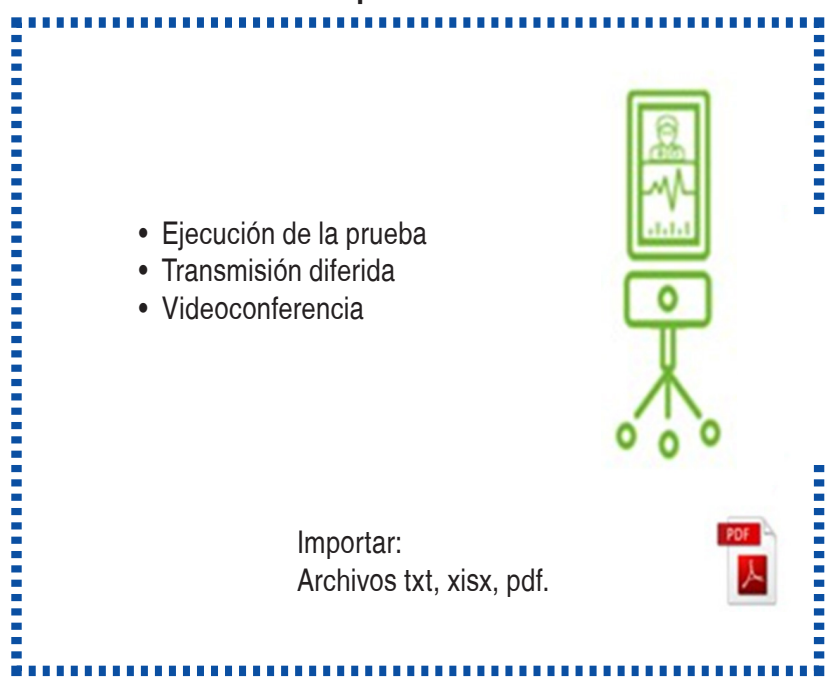

Instituto Nacional de

Enfermedades

Respiratorias



Figura 2: Proceso de referencia-contrarreferencia a distancia de espirometrías (Teleespirometría. Proyecto RENACE).

algunos profesionales en las TIC, falta de conexión, falta de evidencia costo-efectividad y opacidad en los temas legales que conlleva la utilización. ${ }^{29}$

La telemedicina se considera como una herramienta necesaria en lo que se llama el «hospital del futuro». ${ }^{30} \mathrm{En}$ el Instituto Nacional de Enfermedades Respiratorias Ismael Cosío Villegas (INER), Ciudad de México, desde 2015 se trabaja de forma conjunta con el Centro Nacional de Programas Preventivos y Control de Enfermedades (CENAPRECE), la Sociedad Mexicana de Neumología y Cirugía de Tórax (SMNYCT) y con apoyo de la industria farmacéutica para lograr establecer una Red Nacional de Espirometrías (RENACE) que se encuentra en proceso de consolidación en los 32 estados de la República Mexicana. La espirometría es una herramienta esencial para realizar el diagnóstico de enfermedades pulmonares obstructivas como asma y EPOC que no se había podido establecer como medida de uso diario en el primer nivel de atención. ${ }^{31}$

RENACE tiene como objetivos principales dotar de equipos de espirometría a los centros de primer nivel de atención, capacitar al personal de dichos centros en la ejecución de la prueba y verificar la calidad de las espirometrías realizadas en cada uno de los centros espirométricos, así como orientar al personal sobre los posibles diagnósticos y tratamientos en casos específicos (Figura 2).

En relación a la teleeducación, el INER siendo el centro de referencia de enfermedades respiratorias más grande del país, ha incrementado la difusión del conocimiento sobre estos temas mediante la transmisión en vivo de diferentes eventos académicos a través de un canal gratuito y público.
Así, más de 60\% de los asistentes en 2019 han optado por la modalidad en línea (Figura 3).

Es importante que a través de la SMNYCT se apoye y coordine estas iniciativas para poder difundir a las diferentes unidades hospitalarias y universidades los diferentes programas de formación del uso adecuado de la cibersalud con enfoque a las enfermedades respiratorias. Mismo que ya se ha hecho en otras regiones, tales como Australia y Canadá que son ejemplos a seguir. ${ }^{32,33}$

La cibersalud permite que otras áreas afines a la Medicina puedan realizar su labor como: entrevistas de

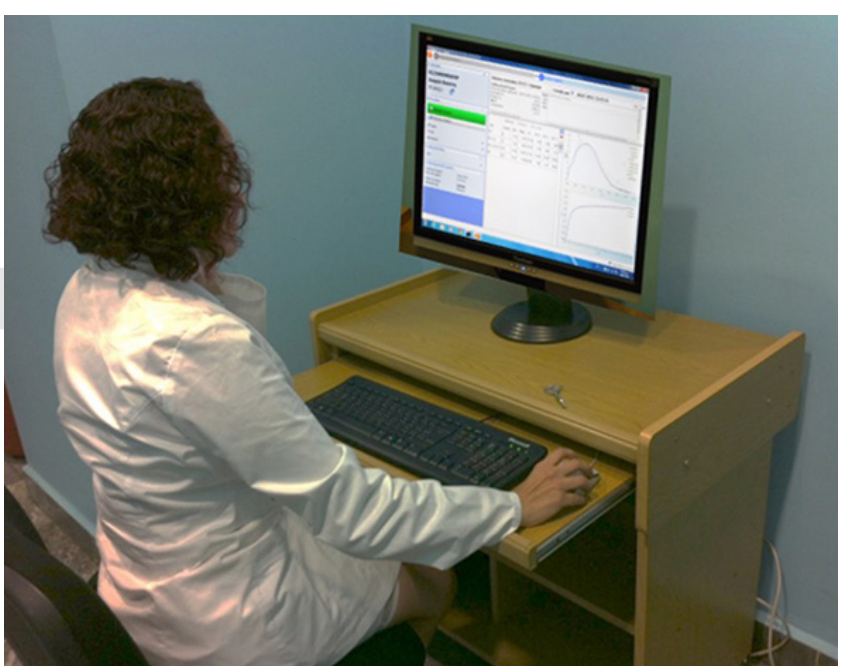

Figura 3: Teleespirometría en el programa RENACE-INER-SMNYCT. 
trabajo social, apoyo psicológico/psiquiátrico, valoración de enfermería para el manejo de heridas y dispositivos, apoyo administrativo dando seguimiento a los diferentes procedimientos institucionales y/o legales, apoyo religioso, promoción de la salud, cumplimiento de programas de epidemiología/investigación, etcétera.

En Europa existe una comisión para que los estados miembros consoliden un sistema de telemedicina para $2020 .^{34}$ Hasta hace una década más de $50 \%$ de los artículos publicados sobre telemedicina se originaban en Estados Unidos, ${ }^{35}$ esto debido al avance tecnológico de dicha región. Hoy en día, las TIC se han globalizado, lo que brinda la posibilidad que países como el nuestro y en la zona latinoamericana se emprenda una nueva cruzada de atención oportuna mediante este mecanismo, ya que las necesidades no cumplidas de la formación de más especialistas, la construcción de clínicas y hospitales faltantes no se visualiza como algo a realizar en el corto plazo.

Actualmente las vías informales de comunicación por los diferentes dispositivos móviles a través de la mensajería denominada WhatsApp son una fuente promisoria de comunicación entre profesionales, pacientes y población general. Hoy WhatsApp se considera como una herramienta valiosa en el ciclo de la telesalud..$^{36}$ Sin embargo, en la construcción de la infraestructura y los procesos para la cibersalud se debe tomar en cuenta algunas consideraciones, más allá del hecho de tener la tecnología para poder hacerlo. Dentro de estas consideraciones existen seis áreas de mayor importancia que debemos definir en el proceso: 1) privacidad del paciente, 2) responsabilidad legal y médica, 3) sustentabilidad, 4) balance de vida del proveedor médico, 5) consideraciones éticas y 6) ciberseguridad.

La privacidad del paciente en el tiempo de la cibersalud es un tema complejo. Los programas deben basarse en reglas básicas en el manejo de la información del paciente. Estos códigos de conducta y expectativas básicas permiten establecer confianza en el sistema. El consultorio médico provee un área donde los pacientes reciben protección de su información, este sistema abre las puertas del consultorio, y con ello el riesgo de infringir o diseminar información sensible.

La responsabilidad médica y legal debe de legislarse de primera instancia mientras construimos redes con mayor interconectabilidad. Conforme la telesalud se expande se generan vulnerabilidades legales para el médico y el paciente. Más aún, abren potenciales retos a través de fronteras. Los mecanismos para asegurar que los proveedores que tienen acceso al sistema cuenten con las credenciales y certificaciones apropiadas para el adecuado funcionamiento del mismo.

La sustentabilidad de las redes es esencial. Como proyecto de un país ciberconectado se deben definir planes que ayuden a limitar el costo y manutención del sistema. Sistemas comunes favorecen la interconectabilidad, pero los hacen más vulnerables a fallos o problemas con proveedores. Para lograr un sistema eficiente hay que pensar en una plataforma que permita el acceso seguro a un portal en el que exista la información relevante para el cuidado del paciente; por lo que esto requiere de una cuidadosa planeación.

La disponibilidad de acceso al sistema comienza a erosionar las fronteras habituales que se establecen para que los proveedores de salud mantengan un balance de vida (horarios de oficina, vacaciones). Al establecer límites y expectativas desde un inicio se ayuda al mejor funcionamiento de los sistemas y su adopción, de esta forma protegemos la calidad de vida del proveedor.

Aún existen consideraciones éticas/bioéticas por anali$z^{2}{ }^{37}$ ya que es posible ofrecer no sólo consulta médica, sino también cirugías a distancia. ${ }^{38}$ Recordando que cualquier aplicación de la telemedicina pudiera ser considerado un acto médico, debe tomarse en cuenta que la consulta a distancia pudiera no ser suficiente en algunos casos para alcanzar los estándares de calidad deseados.

La ciberseguridad es un tema central en cualquier sistema de cibersalud. Conforme crezca nuestra dependencia en los sistemas de cibersalud para proveer de mejores servicios a la población, la construcción de estos proyectos tendrá que proyectar la continua inversión en protección de los sistemas contra ataques del sistema (referencia a la invasión del sistema de salud de Inglaterra). ${ }^{39}$

Todas estas consideraciones hacen más evidente la necesidad para incorporar entrenamiento específico en cibersalud en el currículo de entrenamiento de todos los profesionales de salud. Más aún, es necesaria la educación especializada en cibersalud, para así proveer al sistema nacional de salud con personal médico que entienda las esferas médicas y cibernéticas para mejorar y diseñar nuestros sistemas.

El panorama es amplio, por lo que es necesario que se priorice en los nuevos proyectos nacionales de salud en las bondades de la telemedicina. El advenimiento de la tecnología siempre ha sido una gran aliada al campo del correcto cuidado de los pacientes y la facilitación del quehacer del médico.

\section{REFERENCIAS}

Alaneir DFDS. Conceptualización de la esfera de la telesalud. El desarrollo de la telesalud en América Latina. Santiago de Chile: CEPAL; 2013.p. 141-145.

2. OMS. Cibersalud [Internet]. $58^{\text {a }}$. Asamblea Mundial de la Salud; del 16 al 25 de mayo del 2005; Ginebra (Suiza). Ginebra Suiza: OMS; 2005 (resolución WHA58.28). Fecha de consulta: 25 de julio de 2019. Accesible en: http://apps.who.int/gb/ebwha/pdf_files/WHA58/WHA58_28-sp.pdf.

3. Weinstein RS, Krupinski EA, Doarn CR. Clinical examination component of telemedicine, telehealth, mHealth, and connected health medical practices. Med Clin North Am 2018;102(3):533-544. doi: 10.1016/j.mcna.2018.01.002.

4. Serie Tecnologías en Salud. 2da. ed. Vol. 3. México D.F.; 2011: Centro Nacional de Excelencia Tecnológica en Salud. 
Neumol Cir Torax. 2020; 79 (1): 12-16

5. Sandoval-Gutiérrez JL, Reyes ES, Bautista EB. Pulmonary diseases: first cause of mortality in the world. Chest 2011;139(6):1550. https:/l doi.org/10.1378/chest.11-0021.

6. Pérez-Padilla JR. Muertes respiratorias en México, 2015. Neumol Cir Torax 2018;77(3):198-202.

7. Vázquez-García JC. Crecimiento y distribución geográfica de los neumólogos en México: Implicaciones para el mercado de trabajo y la formación de recursos humanos. Neumol Cir Torax 2010;69(2):64-74.

8. Ambrosino N, Makhabah DN, Sutanto YS. Tele-medicine in respiratory diseases. Multidiscip Respir Med 2017;12:9. https://doi.org/10.1186/ s40248-017-0090-7.

9. Vitacca M, Montini A, Comini L. How will telemedicine change clinical practice in chronic obstructive pulmonary disease? Ther Adv Respir Dis 2018;12:1753465818754778. https://doi.org/10.1177/1753465818754778.

10. Hui CY, Walton R, McKinstry B, Jackson T, Parker R, Pinnock H. The use of mobile applications to support self-management for people with asthma: a systematic review of controlled studies to identify features associated with clinical effectiveness and adherence. J Am Med Inform Assoc 2017;24(3):619-632. https://doi.org/10.1093/jamia/ocw143.

11. McLean G, Murray E, Band R, et al. Interactive digital interventions to promote self-management in adults with asthma: systematic review and meta-analysis. BMC Pulm Med 2016;16(1):83.https://doi. org/10.1186/s12890-016-0248-7.

12. Coma-Del-Corral MJ, Alonso-Álvarez ML, Allende M, et al. Reliability of telemedicine in the diagnosis and treatment of sleep apnea syndrome. Telemed JE Health 2013;19(1):7-12. https://doi.org/10.1089/tmj.2012.0007.

13. Garuti G, Bagatti S, Verucchi E, et al. Pulmonary rehabilitation at home guided by telemonitoring and access to healthcare facilities for respiratory complications in patients with neuromuscular disease. Eur J Phys Rehabil Med 2013;49(1):51-57.

14. Ambrosino N, Vitacca M, Dreher M, etal. Tele-monitoring of ventilatordependent patients: a European Respiratory Society Statement. Eur Respir J 2016;48(3):648-663. doi: 10.1183/13993003.01721-2015.

15 Lundell S, Holmner A, Rehn B, Nyberg A, Wadell K. Telehealthcare in COPD: a systematic review and meta-analysis on physical outcomes and dyspnea. Respir Med 2015;109(1):11-26. https://doi.org/10.1016/j. rmed.2014.10.008.

16. Burgos F, Disdier C, de Santamaria EL, et al. Telemedicine enhances quality of forced spirometry in primary care. Eur Respir J 2012;39(6):1313-1318. DOI: 10.1183/09031936.00168010.

17. Segrelles Calvo G, Gómez-Suárez C, Soriano JB, etal. A home telehealth program for patients with severe COPD: The PROMETE study. Respir Med 2014; 108(3):453-462. https://doi.org/10.1016/j.rmed.2013.12.003.

18. Vitacca M, Bianchi L, Guerra A, et al. Tele-assistance in chronic respiratory failure patients: a randomised clinical trial. Eur Respir J 2009;33(2):411-418. doi: 10.1183/09031936.00005608.

19. Vitacca M, Paneroni M, Grossetti F, Ambrosino N. Is there any additional effect of tele-assistance on long-term care programmes in hypercapnic COPD patients? A retrospective study. COPD 2016;13(5):576-582. https://doi.org/10.3109/15412555.2016.1147542.

20. Shany T, Hession M, Pryce D, et al. A small-scale randomised controlled trial of home telemonitoring in patients with severe chronic obstructive pulmonary disease. J Telemed Telecare 2017;23(7):650656. https://doi.org/10.1177/1357633X16659410

21. Vogelmeier CF, Criner GJ, Martinez FJ, et al. Global strategy for the diagnosis, management, and prevention of chronic obstructive lung disease 2017 Report: GOLD executive summary. Eur Respir J 2017;49(3). pii: 1700214. DOI: 10.1183/13993003.00214-2017.

22. Vasilopoulou M, Papaioannou Al, Kaltsakas G, Louvaris Z, Chynkiamis N, Spetsioti S, et al. Home-based maintenance tele- rehabilitation reduces the risk for acute exacerbations of COPD, hospitalizations and emergency department visits. Eur Respir J 2017;49(5). pii: 1602129. DOl: 10.1183/13993003.02129-2016.

23. Robson KS, West AJ. Improving survival outcomes in lung transplant recipients through early detection of bronchiolitis obliterans: daily home spirometry versus standard pulmonary function testing. Can J Respir Ther 2014;50(1):17-22.

24. Telesalud. Fecha de consulta: 15 de marzo 2019. Accesible en: http:// www.cenetec.salud.gob.mx/contenidos/telesalud/telesalud.html.

25. Telemedicina. Fecha de consulta: 15 de marzo de 2019. Accesible en: https://www.blumedica.mx/telemedicina/.

26. Avanza el uso de la telesalud o telemedicina en México. Fecha de consulta 15 de marzo 2019. Accesible en: https://www.gob.mx/salud/ prensa/avanza-el-uso-de-la-telesalud-o-telemedicina-en-mexico.

27. IMSS va por médico en tu casa. Clínicas nocturnas y telemedicina. Fecha de consulta: 15 de marzo de 2019. Accesible en: https://www. excelsior.com.mx/nacional/imss-va-por-medico-en-tu-casa-clinicasnocturnas-y-telemedicina/ 1302055.

28. Millennials, los nuevos players de la salud digital. Fecha de consulta: 15 de marzo de 2019. Accesible en: https://www.saludiario.com/ millennials-los-nuevos-players-de-la-salud-digital/.

29. Vagheggini G, Mazzoleni S, Ambrosino N. Tele-assistance in pulmonary diseases: current status and open issues. SOB 2013;2:80-83.

30. El futuro (tecnológico) de la salud: robots cirujanos y consultas por videollamada. Fecha de consulta: 15 de marzo de 2019. Accesible en: https://www.eldiario.es/hojaderouter/tecnologia/telemedicinacirugia_robotica-salud-tecnologia_0_654785081.html.

31. Historia de la espirometría. Fecha de consulta: 15 de marzo de 2019. Accesible en: https://articulos.sld.cu/neumologia/2013/03/14/historiade-la-espirometria/.

32. Guill MA. National Telehealth Strategy For Australia- For Discussion. Access date: 2019 March 15. Available from: https://www.who.int/goe/ policies/countries/aus__support_tele.pdf.

33. Owens B. Telemedicine on the rise but lagging in Canada. Access date: 2019 March 15. Available from: http://www.cmaj.ca/content/190/38/E1149.

34. Hernandez C, Mallow J, Narsavage GL. Delivering telemedicine interventions in chronic respiratory disease. Breathe (Sheff) 2014;10(3):199-212.DOI: 10.1183/20734735.008314.

35. Cooper CB. Respiratory applications of telemedicine. Thorax 2009;64(3):189-191. doi: 10.1136/thx.2008.104810.

36. Giordano V, Koch H, Godoy-Santos A, Dias Belangero W, Esteves Santos Pires R, Labronici P. WhatsApp messenger as an adjunctive tool for telemedicine: an overview. Interact J Med Res 2017;6(2): e11. doi: 10.2196/jimr.6214.

37. Ali J, Labrique $\mathrm{AB}$, Gionfriddo $\mathrm{K}$, et al. Ethics considerations in global mobile phone-based surveys of noncommunicable diseases: a conceptual exploration. J Med Internet Res 2017;19(5): e110. do: 10.2196/jmir.7326.

38. George El, Brand TC, LaPorta A, Marescaux J, Satava RM. Origins of robotic surgery: from skepticism to standard of care. JSLS 2018;22(4). pii: e2018.00039. doi: 10.4293/JSLS.2018.00039. DOI: 10.4293/ JSLS.2018.00039.

39. Ghafur S, Grass E, Jennings NR, Darzi A. The challenges of cybersecurity in health care: the UK National Health Service as a case study. The Lancet Digital Health 2019;1(1):e10-e12. https://doi. org/10.1016/S2589-7500(19)30005-6.

Conflicto de intereses: Los autores declaran no tener conflicto de intereses. 